\title{
Liquid-crystal patterns of rectangular particles in a square nanocavity
}

\author{
Miguel González-Pinto* \\ Departamento de Física Teórica de la Materia Condensada, Facultad de Ciencias, Universidad Autónoma de Madrid, \\ E-28049 Madrid, Spain
}

Yuri Martínez-Ratón ${ }^{\dagger}$

Grupo Interdisciplinar de Sistemas Complejos, Departamento de Matemáticas, Escuela Politécnica Superior, Universidad Carlos III de Madrid, Avenida de la Universidad 30, 28911 Leganés, Madrid, Spain

Enrique Velasco

Departamento de Física Teórica de la Materia Condensada and Condensed Matter Physics Center, Facultad de Ciencias, Universidad Autónoma de Madrid, E-28049 Madrid, Spain

(Received 12 July 2013; published 23 September 2013)

\begin{abstract}
Using density-functional theory in the restricted-orientation approximation, we analyze the liquid-crystal patterns and phase behavior of a fluid of hard rectangular particles confined in a two-dimensional square nanocavity of side length $H$ composed of hard inner walls. Patterning in the cavity is governed by surface-induced order as well as capillary and frustration effects and depends on the relative values of the particle aspect ratio $\kappa \equiv L / \sigma$, with $L$ the length and $\sigma$ the width of the rectangles $(L \geqslant \sigma)$, and cavity size $H$. Ordering may be very different from bulk $(H \rightarrow \infty)$ behavior when $H$ is a few times the particle length $L$ (nanocavity). Bulk and confinement properties are obtained for the cases $\kappa=1,3$, and 6. In bulk the isotropic phase is always stable at low packing fractions $\eta=L \sigma \rho_{0}$ (with $\rho_{0}$ the average density) and nematic, smectic, columnar, and crystal phases can be stabilized at higher $\eta$ depending on $\kappa$ : For increasing $\eta$ the sequence of isotropic to columnar is obtained for $\kappa=1$ and 3, whereas for $\kappa=6$ we obtain isotropic to nematic to smectic (the crystal being unstable in all three cases for the density range explored). In the confined fluid surface-induced frustration leads to fourfold symmetry breaking in all phases (which become twofold symmetric). Since no director distortion can arise in our model by construction, frustration in the director orientation is relaxed by the creation of domain walls (where the director changes by $90^{\circ}$ ); this configuration is necessary to stabilize periodic phases. For $\kappa=1$ the crystal becomes stable with commensurate transitions taking place as $H$ is varied. These transitions involve structures with different number of peaks in the local density. In the case $\kappa=3$ the commensurate transitions involve columnar phases with different number of columns. In the case $\kappa=6$ the high-density region of the phase diagram is dominated by commensurate transitions between smectic structures; at lower densities there is a symmetry-breaking isotropic to nematic transition exhibiting nonmonotonic behavior with cavity size. Apart from the present application in a confinement setup, our model could be used to explore the bulk region near close packing in order to elucidate the possible existence of disordered phases at close packing.
\end{abstract}

DOI: 10.1103/PhysRevE.88.032506

PACS number(s): 61.30.Cz, 61.30.Hn, 61.30.Jf, 82.70.Dd

\section{INTRODUCTION}

Confinement is known to affect the liquid-crystal ordering behavior of a fluid in a dramatic fashion. In fluids with first-order isotropic $(I)$ to nematic $(N), I \rightarrow N$, transitions in bulk, there exists a corresponding $I \rightarrow N$ transition when the fluid is confined in a pore of size $h$. Depending on the nature of the liquid crystal, the transition is shifted by $\Delta T$ in temperature $T$ or by $\Delta \mu$ in chemical potential $\mu$ with respect to the bulk transition, a phenomenon called capillary ordering. This effect is similar to that occurring in normal, isotropic liquids (the so-called capillary condensation) and is governed by the corresponding macroscopic Kelvin equation [1], which predicts $\Delta T$ or $\Delta \mu \sim h^{-1}$ for $h \rightarrow \infty$. The nature of surface interactions determines whether the shift is positive or negative and $O\left(h^{-2}\right)$ and higher-order corrections may be included

\footnotetext{
*miguel.gonzalezp@uam.es

†yuri@math.uc3m.es

‡enrique.velasco@uam.es
}

for not-so-large pores. This phenomenon also applies to liquid-crystalline phases exhibiting partial or full spatial order, such as smectic (Sm), columnar (Col), or crystalline (Cry), provided the bulk transition is of first order and the behavior of the confined transition is described by a corresponding Kelvin equation, valid in the regime of large pores, with corrections due to elasticity of the periodic structure.

Confinement brings about further complex phase behavior due to commensurate effects between the cavity size $h$ and the natural periodicity of the liquid-crystal structure $a$ in the case of partially or fully ordered phases. In some cases the ordered structure can be suppressed altogether when $h \neq n a$, where $n$ is an integer. This effect has been studied in crystals [2-4] and in liquid crystals and similar systems in three [5-9] and two [10] dimensions; in all of these cases, commensurate transitions are observed involving structures with different numbers of unit cells. In the case of Sm phases these transitions are sometimes called layering transitions and are similar to those occurring in adsorption systems since they involve the creation of a new layer in a stepwise manner. In some circumstances the capillary-ordering transition interacts with 

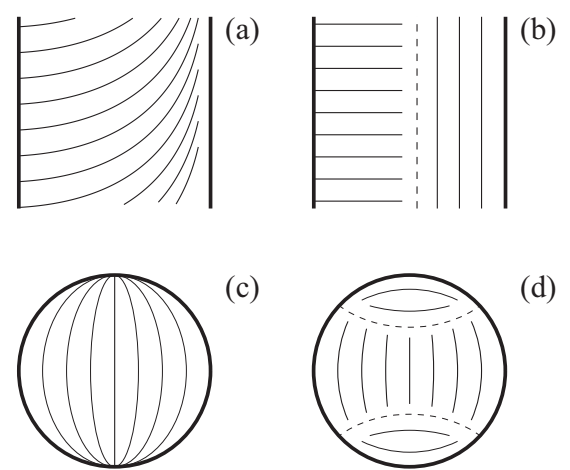

(c)

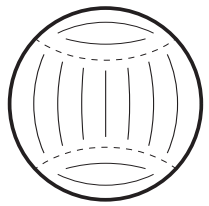

(d)
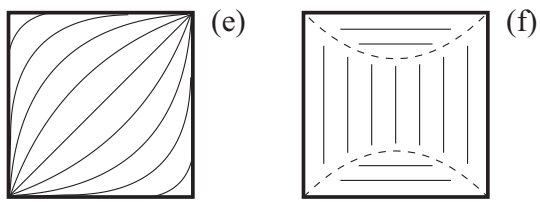

FIG. 1. Schematic of three two-dimensional systems with surface-induced frustration: (a) and (b) planar slit pore with favored surface orientation of the director at right angles, (c) and (d) circular cavity with planar surface orientation, and (e) and (f) square cavity with planar surface orientation. In all cases the surface free energy is satisfied, but in (a), (c), and (e) the director field is distorted with the possible creation of point defects, whereas in (b), (d), and (f) domain walls separating nematic regions with different orientations (dashed lines) are formed.

the commensurate transitions in a complicated manner and for small pore sizes capillary ordering may become intermittent.

In this paper we focus on the additional phenomenology caused by the presence of surface-induced frustration in a fluid confined in a nanocavity, i.e., a cavity a few particle lengths in size. Surfaces are known to be able to orient (anchor) the director of a liquid crystal along specific directions, meaning that the fluid will pay a surface free-energy cost for not choosing the favored alignment at the wall so that any gross misalignment is discouraged (strong anchoring condition). If two regions of the confining surface favor different directions, the liquid crystal will be subject to frustration: Distortion (or elastic) and defect free energies, both of which cannot be optimized at the same time, will compete. In turn, due to capillarity and commensurate effects, the resulting phase behavior, involving typically the formation of defects, may indeed be quite complex.

A simple geometry for the surface-induced frustration effect is a planar slit-pore system with the two walls favoring different alignments, for example, homeotropic $\left(\psi=0^{\circ}\right.$, where $\psi$ is the angle between the nematic director and the surface normal) and planar $\left(\psi=90^{\circ}\right)$. This system is depicted schematically in Figs. 1(a) and 1(b). In the nematic regime, one of two situations may happen: Either the nematic director smoothly rotates between the two surfaces, incurring an elastic free energy [distorted configuration, Fig. 1(a)], or a planar interface separating two films of uniform nematic director with two competing orientations may be formed [Fig. 1(b)], called a steplike phase. A structural transition between the two structures arises as pore size or thermodynamic conditions are varied. This phenomenon was predicted to occur in the region about a line defect [11]. In a slit pore, it was predicted using Landau-de Gennes theory $[12,13]$ and has been further analyzed with the same technique [14] by density-functional theory [15,16] and by simulation [17-19]. The steplike phase is due to strong anchoring conditions of the director at the surfaces together with a large elastic free energy compared to the free energy of formation of step interfaces or walls. A similar phenomenon has been studied, using Monte Carlo simulation, by de las Heras and Velasco [20] in two-dimensional fluids of hard rods confined in circular cavities inducing planar anchoring conditions, where the circular wall induces a distorted-director configuration with two point defects at intermediate densities [Fig. 1(c)]; at higher densities two interfaces separating nematic regions with a slight director distortion result in a more stable configuration [Fig. 1(d)]. In a square cavity the distorted configuration will have four defects at the corners [Fig. 1(e)], which will give rise at higher densities to an undistorted phase with two domain walls [Fig. 1(f)]. A crucial point is that spatially nonuniform phases, such as Sm, Col, and Cry phases, may only form in nanocavities in undistorted-director configurations, so the stabilization of the domain-wall configurations depicted in Figs. 1(b), $1(d)$, and 1(f) is a necessary condition for the formation of these nonuniform phases. Once the domain-wall phases have been stabilized the formation of nonuniform phases and the occurrence of commensurate transitions are possible.

The way some regions of the confined Col, Sm, and Cry phases with different director orientation and/or number of layers grow at the expense of the others when external conditions are changed is an interesting problem. The topology of the free-energy landscape defined by all the local minima (whether stable or not) separated by energy barriers is crucial as it dictates the path the system will follow between two equilibrium states. A study of the dynamical evolution would require one to impose mass conservation to derive the evolution equations for the density profiles. Based on the dynamical and equilibrium density-functional theory, a phasefield liquid-crystal model was recently derived that allows the study of nonequilibrium density and order-parameter dynamic evolutions [21]. However, the evolution that follows from the conjugate-gradient scheme used in the present work to obtain the possible local minima of the grand potential as the state variables are changed will result in a time evolution that resembles the real dynamical evolution. We have used this technique to analyze how the textures of the confined Col, $\mathrm{Sm}$, and Cry phases (depending on the value of particle aspect ratio) evolve between two states with different numbers of cells (columns, layers, or nodes) as the external conditions are varied. The mechanism by which new periodic cells grow depends on the symmetry of the confined phases (particles oriented parallel, Col, or perpendicular, Sm, to the layers), the nature (continuous versus first order) of the bulk phase transitions, and the amount of orientational ordering of the system.

The interest in these studies is also motivated by the possibility that experiments on quasiamonolayers of vibrated granular rods can be related to thermally equilibrated molecular or colloidal fluids of hard anisotropic particles [22-24]. There is ample evidence that these monolayers do form liquidcrystalline phases and surface effects, nematic ordering, etc., 
(a)

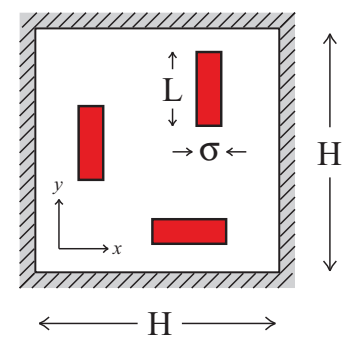

(b)

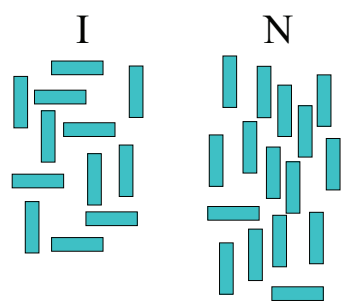

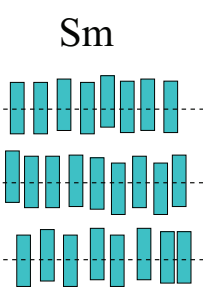

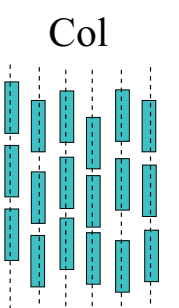

Cry

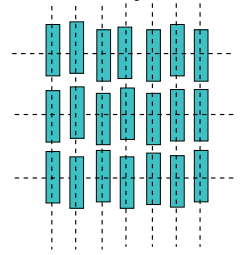

FIG. 2. (Color online) (a) Schematic of the system studied: a fluid of hard rectangles of length $L$ and width $\sigma$ that can orient only along two perpendicular directions $x$ and $y$, inside a square cavity of side length $H$. (b) Sketches of typical particle configurations for the phases obtained in this work: isotropic $(I)$, nematic $(N)$, smectic $(\mathrm{Sm})$, columnar $(\mathrm{Col})$, and crystal (Cry). Dashed lines indicate lattice positions in partially or fully ordered phases.

may be explored in these experimental systems and compared with their thermal counterparts to look for similarities in their ordering behavior, especially considering that overlap or exclusion interactions are the key interactions in both systems.

In this paper we use a simple fundamental-measure density-functional theory to study the ordering properties and thermodynamic behavior of a fluid of hard rectangular particles confined in a square cavity composed of hard inner walls. The model only admits two particle orientations and is unable to describe configurations where the director field is distorted. Since we are interested in the interplay between surface-induced frustration, capillarity, and commensurate effects as far as high-density nonuniform phases are concerned, the nonexistence of the intermediate configuration depicted in Fig. 1(e) is not problematic. The model correctly describes spatial correlations between rectangles in parallel or perpendicular configurations and therefore is expected to give correct predictions on the liquid-crystal patterns and thermodynamic phase behavior of the system at high densities. Irrespective of the length-to-width ratio of the particles, all liquid-crystal structures found have the twofold symmetric configuration depicted in Fig. 1(f), where the different regions are fluid ( $N$ phase) or organized into layers (Sm phase) or columns (Col phase), depending on the elongation of the rectangles; for squares the fourfold symmetry of the cavity is conserved and the high-density phase is a Cry phase on a square lattice. The bulk and confinement phase diagrams were calculated for a number of systems. The high-density regime of the latter are dominated by commensurate transitions between periodic (Sm, Col, or Cry depending on the system) structures involving different numbers of periodic cells. Except for details of the phase diagram and the mechanisms involved in these commensurate transitions, the phase behavior of this fluid under confinement seems to be quite universal.

The paper is arranged as follows. In the following section the particle model and system are introduced. Then, in Sec. III the density-functional theory used is defined and we discuss how the bulk and confined phases are obtained numerically. The results are presented in Sec. IV, which is divided into subsections, one for each fluid considered. Section V is devoted to discussing the limitations of the model, together with some ways to improve the model and extend it to study the pattern growth dynamics associated with the commensurate transitions. Finally, in Sec. VI we summarize the work and present some conclusions.

\section{PARTICLE MODEL AND SYSTEM}

The particle model used is a rectangle of length $L$ and width $\sigma$ [Fig. 2(a)]. The $\sigma$ parameter will be used as the length scale and we adopt the packing fraction $\eta=L \sigma \rho_{0}$ as the scaled density parameter; $\rho_{0}=N / A$ is the mean density ( $N$ is the number of particles and $A$ the area). The size of the particles will be defined in terms of their aspect ratio $\kappa \equiv L / \sigma$. Particles interact with overlap or exclusion interactions [hard rectangle (HR) model], so the temperature $T$ is irrelevant as far as the phase behavior of the system is concerned. In our model we will use the restricted-orientation or Zwanzig approximation, where particles can only point along four directions, say, the $x$ and $y$ axes in the positive and negative directions. At low $\eta$ a fluid of hard rectangles is in the $I$ phase [Fig. 2(b)], with the long axes of particles pointing in all directions with equal probability. As the packing fraction $\eta$ is increased, the fluid becomes oriented [ $N$ phase, see Fig. 2(b)] if $\kappa \gtrsim 3$ (the precise value has not been determined yet). In the high-density regime phases with spatial order [Sm, Col, or Cry; see Fig. 2(b)] can be stabilized. Low values of $\kappa$ favor the Col phase, where particles arrange into columns (consisting of a one-dimensional fluid of particles flowing in the direction of the long particle axes), whereas for higher $\kappa$ the Sm phase (with fluid rows of particles flowing in the direction perpendicular to the long particle axes) tends to be more stable (note that for the special case $\kappa=1$ the $\mathrm{Col}$ and $\mathrm{Sm}$ phases are identical). The stable phase at densities near the close-packing value $\eta=1$ [or $\rho_{0}=(L \sigma)^{-1}$ ] is not known (except for hard squares where a free-energy minimization using a Gaussian parametrization for the local density predicts the Cry phase to be the stable one), but entropic effects might favor a partially fluid (Sm or $\mathrm{Col}$ ) or disordered Cry phase against the expected, fully ordered Cry phase.

Several theoretical $[25,26]$ and computer-simulation $[27,28]$ studies of the HR fluid, in both the restrictedand free-orientation cases, exist. These studies were mainly motivated by the prediction that this fluid can exhibit an exotic nematic phase, the so-called tetratic phase, possessing fourfold rotational symmetry (even though the particles only have twofold symmetry). The tetratic phase, which has been observed in colloidal fluids [29], is present at low values of $\kappa$, but there are indications that tetratic correlations persist up to substantial values of $\kappa$ [30-32]. For large $\kappa$ the usual $I \rightarrow N$ transition is expected at low density. The high-density fluid has only been studied so far for aspect ratios $\kappa=1[27,33]$, 2 [28], and 3 [10]. As mentioned before, the existence of a perfect crystal is questionable because rectangles will perfectly 
pack even in disordered arrangements, so a residual entropy might exist at close packing. Experiments on hard square colloids [34] observe a striking transition from a hexagonal rotator crystal to a rhombic crystal via a first-order transition. Simulations on parallel hard squares [33] obtain a continuous transition to a Cry phase at $\eta=0.79$. A free minimization of the fundamental-measure density-functional theory for hard squares [35] (identical to that used in the present work) gives a $\mathrm{Col} \rightarrow$ Cry first-order transition at $\eta \sim 0.73$; both phases bifurcate from the $I$ branch at $\eta=0.538$. In the case $\kappa=2$ simulations have shown the possibility that the high-density phase consists of a nonperiodic, random tetratic, possibly glassy phase with a residual entropy of $1.79 k$ per particle (where $k$ is Boltzmann's constant). For $\kappa=3$ the observed high-density phase in the density-functional study of parallel HR by Martínez-Ratón [10] is the Col phase. Columnarlike layering was observed in simulations of a HR fluid confined in a two-dimensional slit pore [36] and layering transitions between Col phases with different numbers of layers were obtained in Ref. [10] using density-functional theory.

\section{FREE-ENERGY FUNCTIONAL}

The free-energy density functional used is a fundamentalmeasure functional for hard rectangles where only two particle orientations, along $x$ and $y$, are possible. This can be mapped onto the equivalent problem of a mixture of two components of local densities $\rho_{\nu}(\boldsymbol{r})$, with $v=\{x, y\}$ and where $\boldsymbol{r}$ refers to the position of the particle center of mass. As usual, the Helmholtz free-energy functional $\mathcal{F}\left[\rho_{\nu}\right]=\mathcal{F}_{\text {id }}\left[\rho_{\nu}\right]+\mathcal{F}_{\text {ex }}\left[\rho_{\nu}\right]$ is split into ideal $\mathcal{F}_{\text {id }}\left[\rho_{\nu}\right]$ and excess $\mathcal{F}_{\text {ex }}\left[\rho_{\nu}\right]$ parts, with

$$
\beta \mathcal{F}_{\mathrm{id}}\left[\rho_{\nu}\right]=\sum_{\nu} \int_{A} d \boldsymbol{r} \rho_{\nu}(\boldsymbol{r})\left\{\ln \left[\rho_{\nu}(\boldsymbol{r}) \Lambda_{v}^{3}\right]-1\right\},
$$

where $\beta=1 / k T, T$ is the temperature, $A$ is the system area, and $\Lambda_{v}$ is the thermal wavelength of the $v$ th species. In fundamental-measure theory, the excess free-energy functional is written as $\beta \mathcal{F}_{\text {ex }}\left[\rho_{\nu}\right]=\int_{A} d \boldsymbol{r} \Phi(\boldsymbol{r})$, where $\Phi(\boldsymbol{r})$ is the following local free-energy density [37]:

$$
\Phi(\boldsymbol{r})=-n_{0}(\boldsymbol{r}) \ln \left[1-n_{2}(\boldsymbol{r})\right]+\frac{n_{1 x}(\boldsymbol{r}) n_{1 y}(\boldsymbol{r})}{1-n_{2}(\boldsymbol{r})} .
$$

Here $n_{\alpha}(\boldsymbol{r})$ are weighted densities defined as convolutions:

$$
n_{\alpha}(\boldsymbol{r})=\sum_{v} \int_{A} d \boldsymbol{r}^{\prime} \rho_{\nu}\left(\boldsymbol{r}^{\prime}\right) \omega_{\nu}^{(\alpha)}\left(\boldsymbol{r}-\boldsymbol{r}^{\prime}\right)
$$

The weighting functions $\omega_{v}^{(\alpha)}(\boldsymbol{r})$ are particle geometrical measures related to the corners, edge lengths, and surface of a rectangle:

$$
\begin{aligned}
\omega_{v}^{(0)}(\boldsymbol{r}) & =\frac{1}{4} \delta\left(\frac{\sigma_{x}^{\nu}}{2}-|x|\right) \delta\left(\frac{\sigma_{y}^{\nu}}{2}-|y|\right), \\
\omega_{v}^{(1 x)}(\boldsymbol{r}) & =\frac{1}{2} \Theta\left(\frac{\sigma_{x}^{\nu}}{2}-|x|\right) \delta\left(\frac{\sigma_{y}^{\nu}}{2}-|y|\right), \\
\omega_{v}^{(1 y)}(\boldsymbol{r}) & =\frac{1}{2} \delta\left(\frac{\sigma_{x}^{\nu}}{2}-|x|\right) \Theta\left(\frac{\sigma_{y}^{\nu}}{2}-|y|\right), \\
\omega_{v}^{(2)}(\boldsymbol{r}) & =\Theta\left(\frac{\sigma_{x}^{\nu}}{2}-|x|\right) \Theta\left(\frac{\sigma_{y}^{\nu}}{2}-|y|\right),
\end{aligned}
$$

with $\delta(x)$ and $\Theta(x)$ the Dirac delta and Heaviside functions, respectively. We have defined $\sigma_{\mu}^{\nu}=\sigma+(L-\sigma) \delta_{\mu \nu}$, with $\delta_{\mu \nu}$ the Kronecker symbol (note that $\sigma_{\mu}^{\mu}=L$ and $\sigma_{\mu}^{\nu}=\sigma$ if $\mu \neq v)$.

The fluid is confined in a hard square cavity of side length $H$, which can be described in terms of an external potential $V_{\text {ext }}^{(v)}(\boldsymbol{r})$. The potential acts as an impenetrable wall on the particles, i.e.,

$$
\beta V_{\mathrm{ext}}^{(v)}(\boldsymbol{r})= \begin{cases}0 & \text { for } \quad \frac{\sigma_{x}^{v}}{2}<x<H-\frac{\sigma_{x}^{v}}{2}, \\ & \frac{\sigma_{y}^{v}}{2}<y<H-\frac{\sigma_{y}^{v}}{2} \\ \infty & \text { otherwise. }\end{cases}
$$

The grand potential of the fluid is then

$$
\Omega\left[\rho_{\nu}\right]=\mathcal{F}\left[\rho_{\nu}\right]-\sum_{\nu} \int_{A} d \boldsymbol{r} \rho_{\nu}(\boldsymbol{r})\left[\mu_{\nu}-V_{\mathrm{ext}}^{(v)}(\boldsymbol{r})\right],
$$

where $\mu_{v}$ is the chemical potential of the $\nu$ th species. The equilibrium state of the system is obtained by minimizing the grand potential at fixed values of $\mu_{\nu}$.

From the equilibrium local densities $\rho_{\nu}(\boldsymbol{r})$ it is possible to define the total local density

$$
\rho(\boldsymbol{r})=\sum_{v} \rho_{v}(\boldsymbol{r})=\rho_{x}(\boldsymbol{r})+\rho_{y}(\boldsymbol{r})
$$

and a local packing fraction $\eta(\boldsymbol{r})=\rho(\boldsymbol{r}) L \sigma$. A local order parameter $Q(\boldsymbol{r})$ can also be defined from the local ordering tensor, with elements $Q_{i j}=\left\langle 2 \hat{\boldsymbol{e}}_{i} \hat{\boldsymbol{e}}_{j}-\delta_{i j}\right\rangle$. Here $\langle\cdots\rangle$ is a thermal average and $\hat{\boldsymbol{e}}$ is the particle long axis, with $i, j=1,2$ denoting the two Cartesian components. Since, in our restricted-orientation model, the local angular distribution function $h(\varphi, r)$ can be written as

$$
\begin{aligned}
h(\varphi, \boldsymbol{r})= & \frac{\rho_{x}(\boldsymbol{r})}{\rho(\boldsymbol{r})}\left[\frac{\delta(\varphi)+\delta(\varphi-\pi)}{2}\right] \\
& +\frac{\rho_{y}(\boldsymbol{r})}{\rho(\boldsymbol{r})}\left[\frac{\delta\left(\varphi-\frac{\pi}{2}\right)+\delta\left(\varphi-\frac{3 \pi}{2}\right)}{2}\right],
\end{aligned}
$$

the elements of the local ordering tensor are

$$
\begin{aligned}
Q_{i j}(\boldsymbol{r}) & =\int_{0}^{2 \pi} d \varphi h(\varphi, \boldsymbol{r})\left(\begin{array}{cc}
\cos 2 \varphi & \sin 2 \varphi \\
\sin 2 \varphi & -\cos 2 \varphi
\end{array}\right) \\
& =Q(\boldsymbol{r})\left(\begin{array}{cc}
1 & 0 \\
0 & -1
\end{array}\right) .
\end{aligned}
$$

Then the local order parameter is

$$
Q(\boldsymbol{r})=\int_{0}^{2 \pi} d \varphi \cos 2 \varphi h(\varphi, \boldsymbol{r})=\frac{\rho_{x}(\boldsymbol{r})-\rho_{y}(\boldsymbol{r})}{\rho(\boldsymbol{r})} .
$$

Clearly $-1 \leqslant Q \leqslant 1$. Since the ordering tensor is always diagonal in the reference frame defined by the two possible perpendicular orientations, in the present model the director can only point along two directions: the $x$ axis $(Q>0)$ or the $y$ axis $(Q<0)$. Intermediate situations with the director pointing at an angle $\varphi$ different from $0^{\circ}, 90^{\circ}, 180^{\circ}$, or $270^{\circ}$ cannot be described. In particular, a nematic film sandwiched between two uniform parallel lines that exert antagonistic boundary conditions [e.g., parallel and homeotropic, Fig. 1(b)] will always develop a step or domain wall since the director cannot rotate to form a linearly rotating director field (the 
stable solution predicted by elasticity theory). Also, distorteddirector configurations such as that represented in Fig. 1(e) for the square cavity will never appear as solutions of the model. Still within a restricted-orientation approximation, at least a few intermediate orientations, e.g., $\varphi=45^{\circ}$ and $135^{\circ}$ (and, due to the particle head-tail symmetry, their equivalent orientations $\varphi=225^{\circ}$ and $315^{\circ}$ ), would be needed to describe such configurations. Since we are mainly interested in the high-density regime of the model, we did not pursue that line here.

\section{A. Bulk nematic phase and $I \rightarrow N$ phase transition}

In the case of a uniform nematic phase, $\rho_{v}(\boldsymbol{r})=\rho_{v}=$ const and the free energy simplifies considerably. Inverting the equations that define $\rho$ and $Q$ in terms of $\rho_{x}$ and $\rho_{y}$, we obtain

$$
\rho_{x}=\frac{\rho}{2}(1+Q), \quad \rho_{y}=\frac{\rho}{2}(1-Q) .
$$

It is easy to obtain the relations $n_{0}=\rho, n_{2}=\eta$, and

$$
\begin{aligned}
& n_{1 x}=\frac{\rho}{2}[L+\sigma+(L-\sigma) Q], \\
& n_{1 y}=\frac{\rho}{2}[L+\sigma-(L-\sigma) Q] .
\end{aligned}
$$

From here, the free-energy density is

$$
\begin{aligned}
\frac{\beta \mathcal{F}}{A}= & \rho\left\{\ln \rho-1+\frac{1}{2}\left[(1+Q) \ln \left(\frac{1+Q}{2}\right)\right.\right. \\
& \left.+(1-Q) \ln \left(\frac{1-Q}{2}\right)\right] \\
& -\ln (1-\eta)+\frac{\eta}{4(1-\eta)}\left[\left(\kappa+\kappa^{-1}+2\right)\right. \\
& \left.\left.-\left(\kappa+\kappa^{-1}-2\right) Q^{2}\right]\right\},
\end{aligned}
$$

where we have defined $\kappa \equiv L / \sigma$ as the aspect ratio of the rectangle (here and in the following the thermal wavelengths $\Lambda_{v}$ are assumed to be absorbed in the chemical potentials $\left.\mu_{v}\right)$. Direct minimization of $\mathcal{F}$ with respect to $Q$ leads to the following transcendental equation:

$$
Q=\frac{1-e^{-y\left(\kappa+\kappa^{-1}-2\right) Q}}{1+e^{-y\left(\kappa+\kappa^{-1}-2\right) Q}},
$$

where $y \equiv \eta /(1-\eta)$. For packing fractions $\eta \geqslant \eta^{*}$ this equation presents a solution $Q \geqslant 0$. The transition can be shown to be continuous. Assuming $Q \ll 1$ and expanding to lowest order, we find $\eta^{*}=2 /\left(\kappa+\kappa^{-1}\right)$. Of course the $I \rightarrow N$ transition is in some cases preempted by a transition to a nonuniform phase, as we show below.

\section{B. Bulk nonuniform phases}

In order to obtain the stability regions where possible nonuniform (Sm, Col, and Cry) phases can be stable, a numerical minimization of the grand potential $\Omega$ at fixed $\mu_{v}$ is required. Assuming a periodic structure in $x$ and $y$ with periods $d_{x}$ and $d_{y}$, respectively, we chose to perform a Fourier expansion of the density profile

$$
\rho_{\nu}(\boldsymbol{r})=\rho_{0} \gamma_{\nu} \sum_{n, m=0}^{\infty} \alpha_{n m}^{(\nu)} \cos \left(n q_{x} x\right) \cos \left(m q_{y} y\right),
$$

where $\rho_{0}$ is the average density, $\gamma_{\nu}$ is the occupancy probability per unit cell of species $v$ (with $\gamma_{x}+\gamma_{y}=1$ ), and $q_{x}=2 \pi / d_{x}$ and $q_{y}=2 \pi / d_{y}$ are wave vectors. The coefficients $\left\{\alpha_{n m}^{(\nu)}\right\}$ are taken as minimization variables together with the periods $d_{x}$ and $d_{y}$ and one of the molar fractions $\gamma_{v}$ and a conjugategradient technique was used to minimize the grand potential. In the case of the $\mathrm{Sm}$ and $\mathrm{Col}$ phases the number of Fourier components can be drastically reduced since we can restrict the indices to the form $\alpha_{n 0}^{(v)}$. In practice, the sums (15) were truncated using the criterion $\left|\alpha_{n m}^{(v)}\right|<10^{-p}$. For $\eta<0.6$ this criterion could be satisfied with $p=8$ and $10 \times 10$ Fourier components; for the highest densities convergence is a bit poorer and $p$ had to be reduced to 5 (with the same number of Fourier components). In all cases an increase in the number of components did not lead to a significant change in the results.

\section{Confined structures}

In the case of the confined system, the grand potential was discretized in real space and the two densities $\rho_{x}$ and $\rho_{y}$ at each grid point were taken as independent variables. The grid step size was $\Delta x=\Delta y=0.05 \sigma$ or 20 points in a particle width $\sigma$ in the cases $\kappa=3$ and 6; in the case $\kappa=1$ a finer grid with $\Delta x=\Delta y=\sigma / 60$ was used. As an example, that gives a grid of $(3 \times 6 \times 20)^{2}=360^{2}$ points in the case $H=3 L$ and $\kappa=6$. Again a conjugate-gradient method, with analytical calculation of the gradient, was used to perform the minimizations. The minimization variables used were not the local densities, but $\sqrt{\rho_{v}(\boldsymbol{r})}$, since these variables show better convergence properties due to their larger variation close to the regions where the density is very small. The iterative process is very efficient and the convergence is such that the absolute value of the conjugate gradient achieved is typically $10^{-12}$ per mesh point. Typically the starting configurations were isotropic configurations $\left(\rho_{x}=\rho_{y}\right)$ at a low value of chemical potential. After equilibration, $\mu$ was increased in small steps, using the converged densities of the previous step. In addition, a series of runs where $\mu$ was decreased at fixed $H$ were performed to search for discontinuous phase transitions. Runs with varying cavity sizes (increasing and decreasing $H$ ) at fixed $\mu$ were also done by changing the cavity size by a few grid points and rescaling the density fields on the grid.

\section{RESULTS}

In this section we show the results of our density-functional investigation on the bulk and confinement phase behavior of the HR fluid. The section is divided into subsections, each including the results for the case $\kappa=1,3$, or 6 .

\section{A. $\kappa=1$}

The bulk phase diagram in this case exhibits the sequence

$$
I \rightarrow \mathrm{Col} \rightarrow \text { Cry }
$$




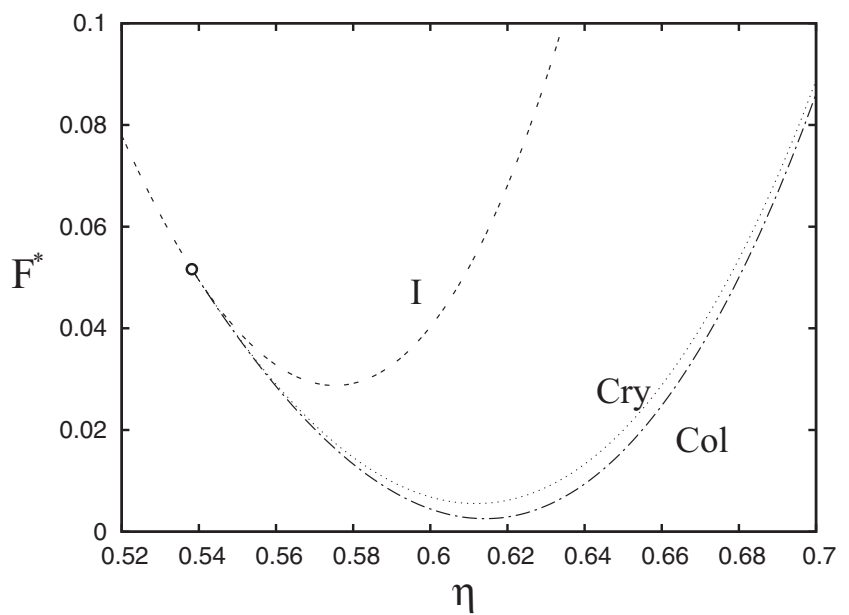

FIG. 3. Free-energy density branches in reduced units $F^{*}=$ $\beta \mathcal{F} L \sigma / A$ of the $I$, Col, and Cry phases with respect to packing fraction for the HR fluid with aspect ratio $\kappa=1$ (hard squares). The straight line $f=a \eta+b$, with $a=6.20$ and $b=-3.22$, has been subtracted from the free energies in order to better visualize the curves. The transition point is located at $\eta=0.538$.

The $I \rightarrow$ Col transition occurs at $\eta=0.538$ and is continuous. The Cry and Col phases have very similar free energies, but a Cry phase with a square-lattice structure becomes more stable at a first-order transition occurring at $\eta \sim 0.750$. (This result has been obtained using a Gaussian parametrization for the local densities; a parametrization-free calculation lowers this number by a few hundredths. Belli et al. [35], using the same density functional, obtain $\eta \sim 0.73$.) Figure 3 shows the free energies of the $I, \mathrm{Col}$, and Cry phases with respect to packing fraction. (Note that, in the case $\kappa=1$, the two species $v=\{x, y\}$ are degenerate and the system can be viewed as a one-component system rather than as mixture; in the present results the former view was adopted, so the free energy and chemical potential do not include the factor $-\ln 2$ from the entropy of mixing.) The Col and Cry free-energy branches bifurcate from the $I$ branch at the same packing fraction (indicated by circles), but the Col phase is more stable up to the transition to the Cry phase. The transition is continuous within the accuracy of our calculations.

In the interval of packing fractions explored here, the Col phase is marginally more stable than the Cry phase in bulk.
However, in the confined fluid, the near degeneracy of the Col and Cry phases in terms of free energy is broken and the Cry phase becomes much more stable; now the restricted geometry and the boundary conditions favor the formation of well-localized density peaks. The reason is the following: Since the cavity has a square shape and particles are completely symmetric (same length and width), the system cannot break the symmetry along a single direction. Even though the four walls could in principle favor Col ordering, this situation obviously generates frustration as particles cannot freely diffuse within each layer due to their orthogonal intersections. Thus the localization of particles on a square lattice minimizes the free energy as the total density is increased.

The number and configuration of the crystal peaks depend very much on the cavity side length $H$. If $a$ is the lattice parameter of the Cry phase (square lattice), we expect $a \simeq$ $1.18 \mathrm{~L}$ in bulk (the prefactor does have a slight dependence on chemical potential). Then a square lattice with $n^{2}$ peaks ( $n$ being an integer) will fit into the cavity when the cavity side is $H=H_{n}$, with $H_{n}=(n-1) a+L$ or $H_{n} / L \simeq 1.18 n-0.18$. Therefore, we expect a transition between a structure with $n^{2}$ peaks, labeled $\mathrm{Cry}_{n}$, and another one with $(n+1)^{2}$ peaks, Cry $_{n+1}$, at roughly $H / L \simeq\left(H_{n}+H_{n+1}\right) / 2 L=1.18 n+0.41$, i.e., at $H / L=1.59,2.77,3.95,5.13,6.31,7.49,8.67, \ldots$.

This is shown in Fig. 4, where a sequence of structures (globally stable for each $\mathrm{H}$ ) from $\mathrm{Cry}_{6}$ to $\mathrm{Cry}_{7}$ are plotted in local-density contour plots. In this case the transition is located somewhere between Figs. 4(c) and 4(d). At each transition point the two structures that coexist will be slightly distorted: The one with $n^{2}$ peaks will be slightly expanded, whereas that with $(n+1)^{2}$ peaks will be slightly contracted. The peaks of these structures are in fact a bit smeared out about the mean positions. The elastic free-energy cost associated with having a lattice parameter different from that in bulk is the driving mechanism of the commensurate transitions. An animation showing different commensurate phase transitions can be found in Ref. [38].

In Fig. 5 the surface phase diagram is plotted in the $\mu-H$ plane. The diagram covers a sequence of transitions from $\mathrm{Cry}_{4}$ to $\mathrm{Cry}_{9}$. The transitions terminate in critical points, indicated by open circles in the diagram. The sequence of critical points, which are always above the bulk transition (dashed horizontal line in the figure), tends to the bulk I-Col (or I-Cry) (a) $\mathrm{H}=6.6 \mathrm{~L}$

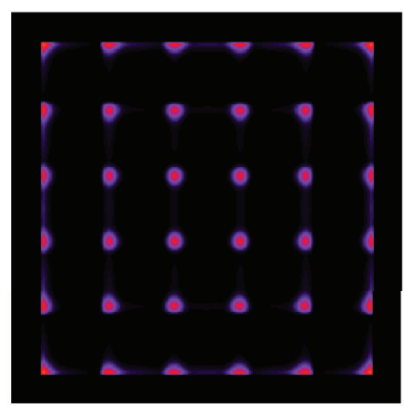

(b) ${ }^{-} \mathrm{H}=7.4 \mathrm{~L}$

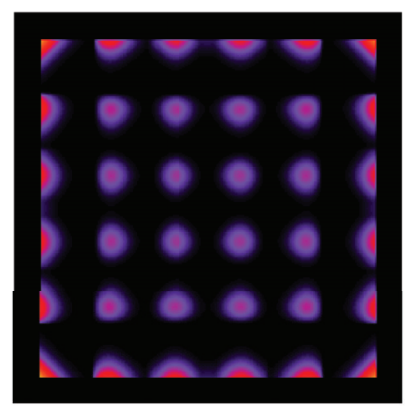

(c) ${ }^{-} \mathrm{H}=7.6 \mathrm{~L}$

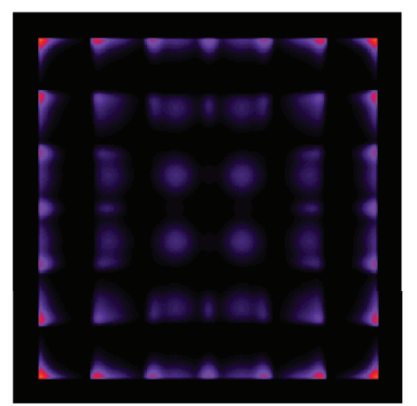

(d) ${ }^{-} \mathrm{H}=\overline{7} .8 \mathrm{~L}$

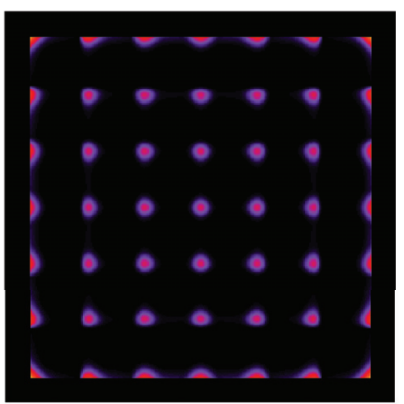

FIG. 4. (Color online) Density contour plots of confined structures in the case $\kappa=1$ and for chemical potential $\beta \mu=8.376$. Different cavity sizes from $H=6.6 \mathrm{~L}$ to $7.8 \mathrm{~L}$ are shown. These structures correspond to the $\mathrm{Cry}_{6} \rightarrow \mathrm{Cry}_{7}$ transition. Brightness saturation has been adjusted differently in each panel to optimize contrast. 


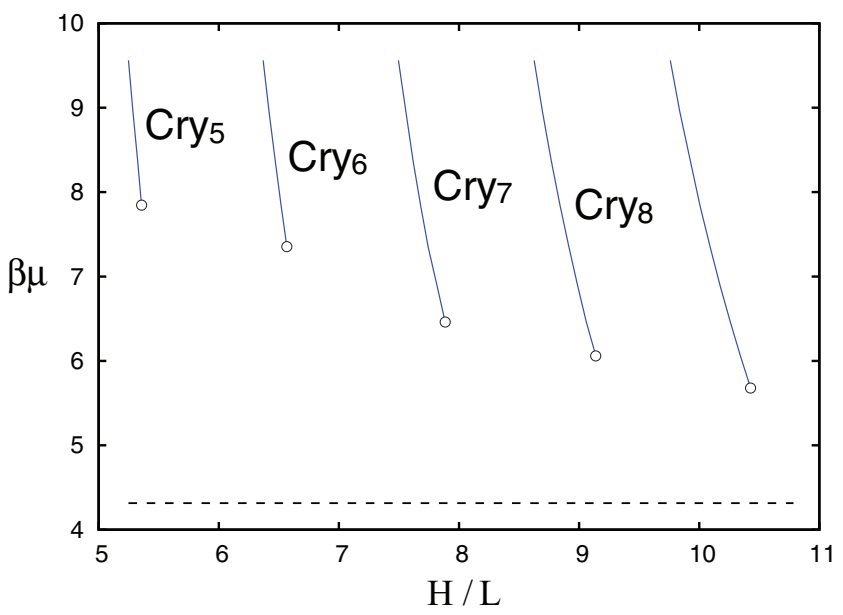

FIG. 5. (Color online) Phase diagram of the HR fluid with aspect ratio $\kappa=1$ (hard squares) in the chemical potential $\mu$ vs cavity lateral size $H$. The open circles indicate the terminal critical points. The dashed horizontal line is the bulk chemical potential of the $I \rightarrow \mathrm{Col}$ transition.

bifurcation value as the cavity size is increased. From a free-energy minimization using a Gaussian parametrization of the density profile, we obtain a bulk Col-Cry transition located at a chemical potential $\beta \mu=10.096$, which is above the range shown in Fig. 5. Also note that, since the bulk transition is continuous, the transition in the cavity is suppressed because the system is confined in both spatial directions and there can be no singularity in the free energy.

$$
\text { B. } \kappa=3
$$

Now the sequence of bulk phases is

$$
I \rightarrow \text { Col. }
$$

The transition is of first order. The other phases, $N, \mathrm{Sm}$, and Cry, are all metastable at least up to $\eta \sim 0.73$ (the maximum density explored). Figure 6 shows the free energies of the different phases, which reflect the first-order character of the $I \rightarrow$ Col transition; the packing fractions of the two coexisting phases are indicated by closed circles. We have not found a stable Cry phase with $\gamma_{x} \neq \gamma_{y}$ and $0<\gamma_{x}, \gamma_{y}<1$ (uniaxial Cry phase); thus the free energy of the Cry phase plotted in Fig. 6 corresponds to that of a one-component fluid of a parallel HR (with $\gamma_{x}=1, \gamma_{y}=0$ or $\gamma_{x}=0, \gamma_{y}=1$ ). This solid is equivalent, after rescaling in the direction of the long rectangular axis, to a system of hard squares. In Ref. [10] a plastic Cry phase with $\gamma_{x}=\gamma_{y}$ was found as a metastable phase using the same model; this branch is not represented here.

The surface properties of this fluid against a hard wall have been investigated in Ref. [10] with the same theoretical model. The preferred orientation of the particles at the wall is parallel. This favors Col-like configurations near the wall and in fact the Col phase wets the wall- $I$ interface, i.e., as the transition density is approached from below, a film of Col phase is adsorbed with a thickness that diverges at the bulk transition. When the system is confined in the cavity, the bulk $I \rightarrow$ Col transition continues as a first-order transition

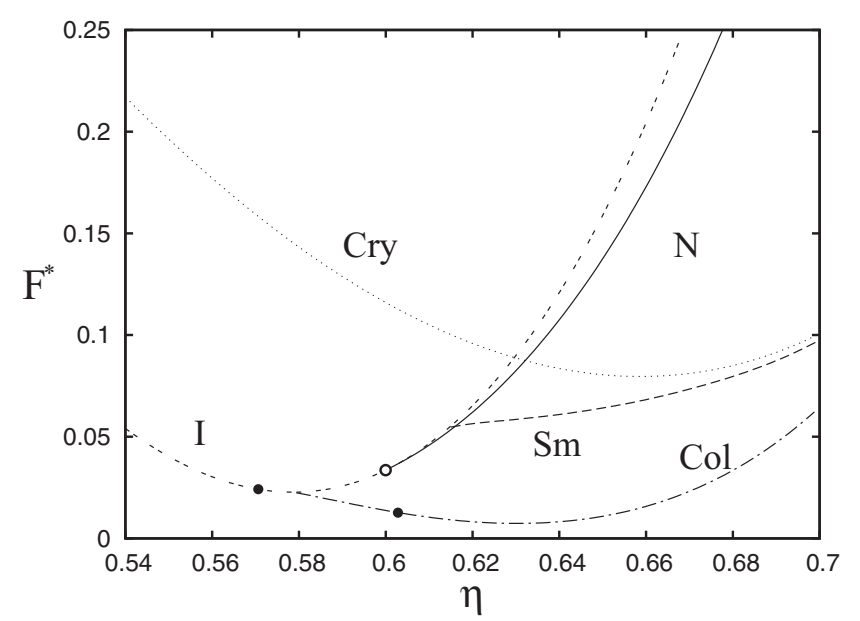

FIG. 6. Free-energy density branches in reduced units $F^{*}=$ $\beta \mathcal{F} L \sigma / A$ of the $I, N, \mathrm{Sm}, \mathrm{Col}$, and Cry phases with respect to packing fraction for the HR fluid with aspect ratio $\kappa=3$. The straight line $f=a \eta+b$, with $a=7.17$ and $b=-3.91$, has been subtracted from the free energies in order to better visualize the curves. The coexistence packing fractions for the $I \rightarrow$ Col transition are $\eta=0.571$ and 0.603 and are represented by closed circles. The bifurcation point for the $I \rightarrow N$ transition (open circle) is at $\eta=0.600$.

at chemical potentials above that of the bulk transition (see Fig. 7). In a wetting situation one would expect the transition to occur below the bulk transition. Here we are dealing with a frustration effect induced by the four surfaces. The transition line becomes a highly nonmonotonic function of cavity size $H$ and in fact is connected to the commensurate transitions that take place between different Col-like structures. In Fig. 8 density false-color plots are shown for different structures along a path at fixed $H=7 L$ that crosses one of the $I \rightarrow \mathrm{Col}$ transition curves. The structure is at first symmetric, but with considerable Col-like oscillations propagating from the four walls. At the transition the symmetry is broken and a well-developed columnar structure (horizontal columns in the

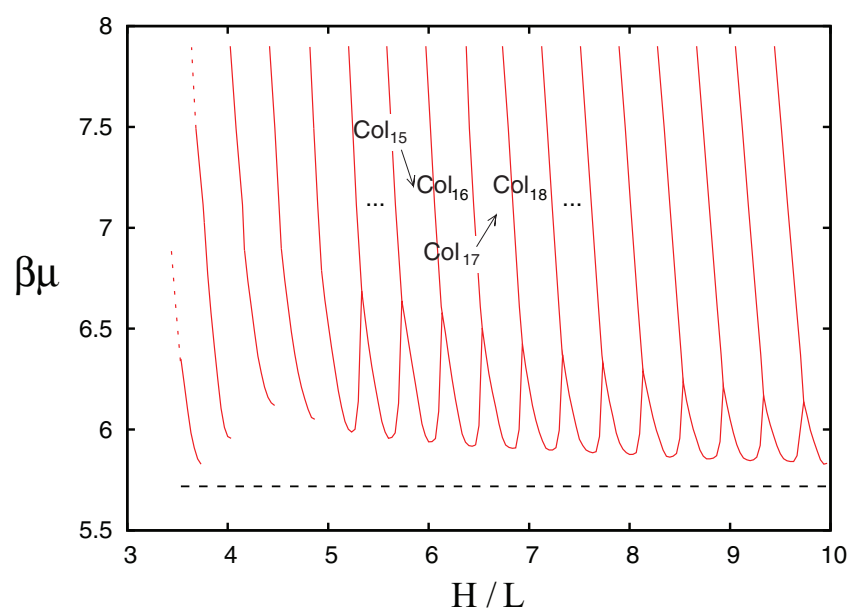

FIG. 7. (Color online) Phase diagram of the HR fluid with aspect ratio $\kappa=3$ in the chemical potential vs. cavity lateral size $H$. The dashed horizontal line is the chemical potential of the first-order bulk $I \rightarrow$ Col transition. 
(a) $\beta \mu=5.28$

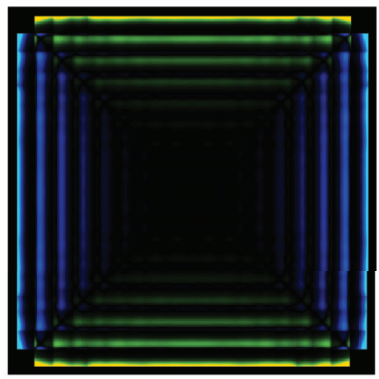

(b) $\beta \mu=5.69$

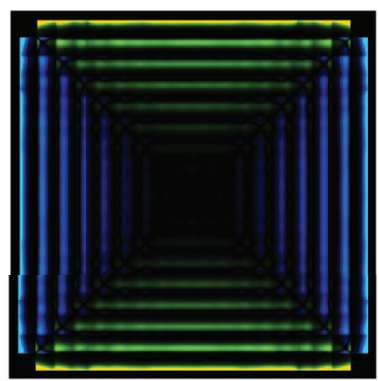

(c) $\beta \mu=6.14$

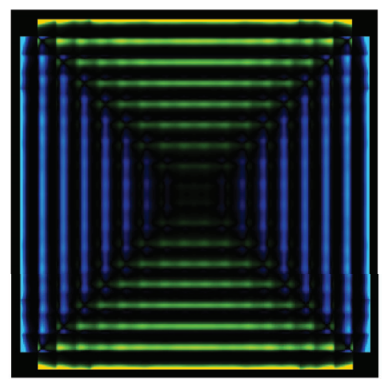

(d) $\beta \mu=6.61$

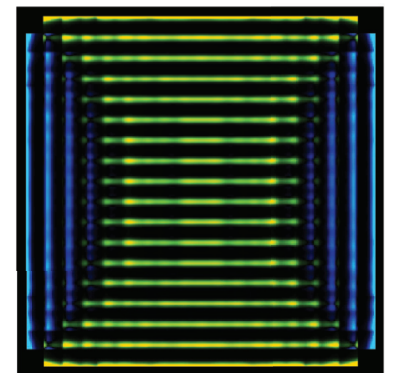

FIG. 8. (Color) Density false-color plots of confined structures in the case $\kappa=3$ for fixed cavity size $H=7 L$. Cases for different chemical potential values (in the range $\beta \mu=5.28-6.61)$ are shown. Note that it is actually the product $Q(\boldsymbol{r}) \rho(\boldsymbol{r})$ [see Eqs. (7) and (10)] that is plotted in the graphs, with the case $\rho_{y}>\rho_{x}$ represented in blue (dark grey, vertical layers) and the case $\rho_{y}<\rho_{x}$ represented in yellow (light grey, horizontal layers).

central region), parallel to two of the surfaces, appears in the cavity, with small islands of columns, in the perpendicular direction, adsorbed on the other two surfaces. The confined Col phase has a complicated structure that results from the inability to satisfy the surface parallel orientation at the four walls of the cavity with a single uniform Col phase. Only two such conditions can be verified and the result is the formation of two regions at two opposing walls where surface orientation is parallel but opposite to that of the central columnarlike region.

The commensurate transitions are of the type $\mathrm{Col}_{n} \rightarrow$ $\mathrm{Col}_{n+1}$, where $\mathrm{Col}_{n}$ is a columnar phase with $n$ columns. At the transition the system develops an additional column in the central region. Because the orientational order is very high, the transition mainly involves translational degrees of freedom in the direction perpendicular to the columns and it is the wall distance along this direction that is relevant. Figure 9 shows a sequence of configurations at high chemical potential, where different confined Col structures are shown in the neighborhood of the $\mathrm{Col}_{24} \rightarrow \mathrm{Col}_{25}$ transition. Figures 9(a)9(c) correspond to the free-energy branch of the $\mathrm{Col}_{24}$ phase. The thermodynamic transition occurs at $H=9.44 L$, so these figures correspond to metastable states. As $H$ increases, the two small regions with columns at perpendicular orientations grow in size at the expense of the central structure, which shrinks and develops highly structured density peaks. Overall the structure gets more symmetric. When the system switches to the $\mathrm{Col}_{25}$ free-energy branch [configuration in Fig. 9(d)], the

peaks in the central region rearrange into a new columnar layer (with the surface structure largely unaffected) and the size of the regions with perpendicular orientation returns to its usual value. Note that, in the stability window of the $\mathrm{Col}_{n}$ phases, these regions look very similar in structure and size regardless of the value of $n$ (for sufficiently large $n$ ). The sequence of configurations shown in Fig. 9 is not necessarily related to the actual kinetic behavior of the phase transition, but gives an indication of how the nucleation of the new phase in the cavity could take place. A film of the evolution of the fluid as the cavity size $H$ is varied can be found in Ref. [38]. Finally, the connection between the commensurate $\mathrm{Col}_{n} \rightarrow \mathrm{Col}_{n+1}$ and capillary $I \rightarrow$ Col transitions is broken for small cavities, which means that the $I$ phase passes continuously into a Col-like phase.

$$
\text { C. } \kappa=6
$$

For this aspect ratio the bulk sequence is very different. Now the Col phase is no longer stable and instead the Sm phase becomes stable. The phase sequence is

$$
I \rightarrow N \rightarrow \mathrm{Sm} \text {. }
$$

Figure 10 presents the free-energy branches of the different phases. The $I \rightarrow N$ transition occurs at $\eta=0.324$ and is continuous. The $N \rightarrow \mathrm{Sm}$ transition is also continuous and takes place at $\eta=0.525$. The Cry phase is unstable at least up to $\eta=0.7$, but then becomes more stable than the $\mathrm{Sm}$

(c) $\mathrm{H}=9.70 \mathrm{~L}$

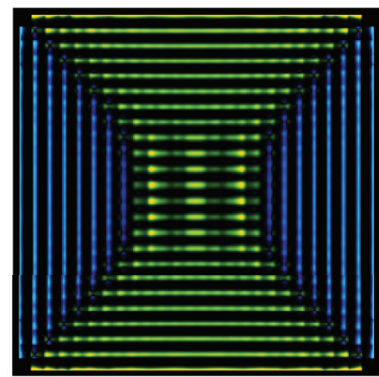

(d) $\mathrm{H}=9.77 \mathrm{~L}$

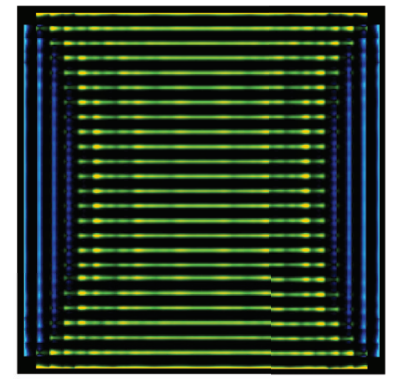

FIG. 9. (Color) Density contour plots of confined structures in the case $\kappa=3$ for $\beta \mu=7.901$, indicating structural changes across one

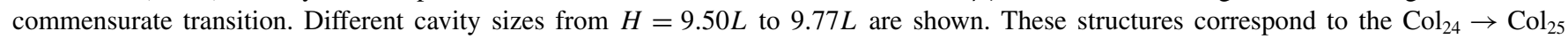
transition. See the caption of Fig. 8 for an explanation of color code. 


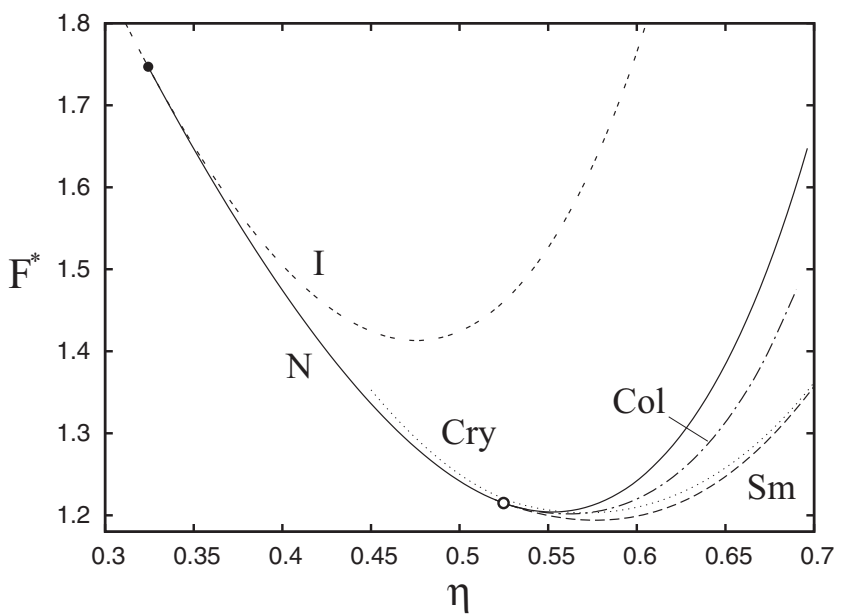

FIG. 10. Free-energy density branches in reduced units $F^{*}=$ $\beta \mathcal{F} L \sigma / A$ of the $I, N, \mathrm{Sm}, \mathrm{Col}$, and Cry phases with respect to packing fraction for the HR fluid with aspect ratio $\kappa=6$. The straight line $f=a \eta+b$, with $a=5.5$ and $b=-4.0$, has been subtracted from the free energies in order to better visualize the curves. The $I \rightarrow N$ transition (closed circle) occurs at $\eta=0.324$, while the $N \rightarrow \mathrm{Sm}$ transition (open circle) is located at $\eta=0.525$.

phase at higher densities. (Again the free-energy density of the Cry phase plotted in Fig. 10 corresponds to a system of parallel HRs, i.e., to a one-component system; since we expect the fraction of the perpendicular species to be negligible in a full calculation, both free energies should be almost identical.) Bifurcation points for the (continuous) $I \rightarrow N$ and $N \rightarrow \mathrm{Sm}$ transitions are indicated by circles. The Sm phase is stable at high densities, but the difference in free energy with the Cry phase decreases, so it is likely that a transition to the crystal takes place at higher densities.

Since the bulk phase diagram of this fluid involves three phases instead of two, the surface phase diagram in the cavity is more complex. Two regions of distinct fluid behavior can be identified; they are covered separately in the following.

\section{1. $I \rightarrow N$ transition under confinement}

Since the bulk $I \rightarrow N$ is continuous, there should be no such transition under confinement. The fact that we do find a

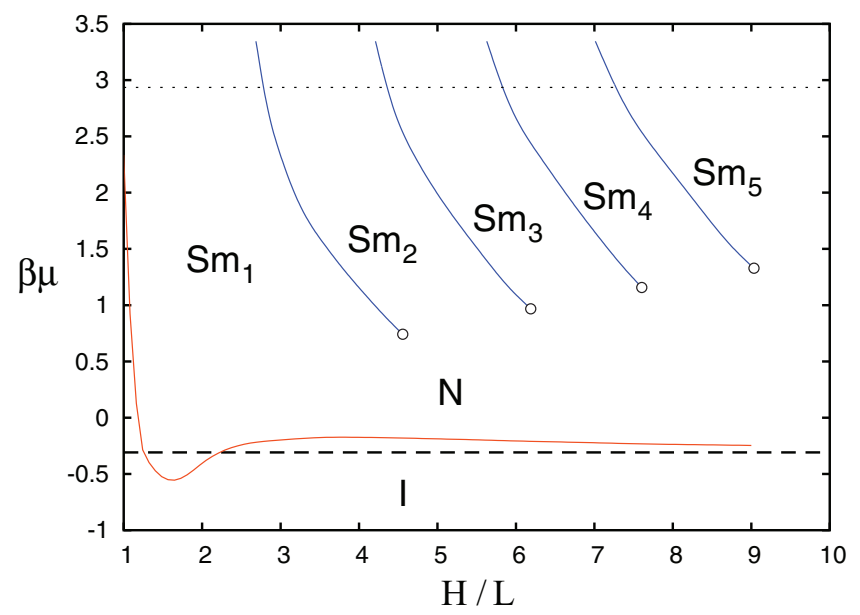

FIG. 12. (Color online) Phase diagram of the HR fluid with aspect ratio $\kappa=6$ in the chemical potential vs cavity lateral size $H$. Open circles are terminal critical points for the commensurate transitions. The dotted horizontal line is the chemical potential of the continuous $N \rightarrow$ Sm transition, while the dashed horizontal corresponds to the continuous bulk $I \rightarrow N$ transition.

transition line in the cavity that connects, for large cavities, with the bulk transition is due to the symmetry breaking of the nematic director inside the square cavity. To see this, we study the evolution of the structure inside the cavity as the density is increased at fixed $H$ from a low value. We refer to Fig. 11, where the order-parameter field is plotted in false color for the case $H=3 L$. At low $\eta$ (or chemical potential $\mu$ ) the fluid is disordered (confined $I$ phase), except at thin regions adsorbed at the four walls where particles are oriented parallel (on average) to the corresponding wall [Fig. 11(a)]. This configuration is fully symmetric since it conforms to the fourfold symmetry of the square cavity. At higher densities the fluid becomes globally oriented and the director breaks the symmetry by choosing one of two possible but equivalent configurations (differing by a global rotation by $90^{\circ}$ ). Figures $11(\mathrm{~b})-11(\mathrm{~d})$ correspond to a choice where the director of the largest nematic region is oriented along the $x$ axis. These twofold symmetric configurations break the fourfold symmetry of the cavity. The broken symmetry has an associated continuous transition, which occurs at a value of chemical potential that tends to that (a) $\beta \mu=-0.39$

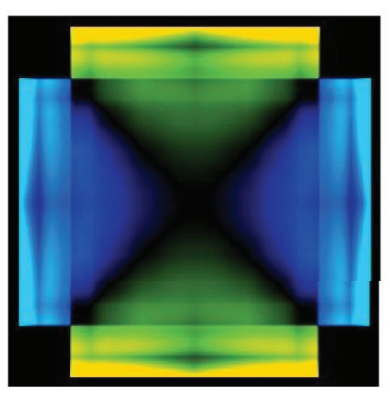

(b) $\beta \mu=-0.15$

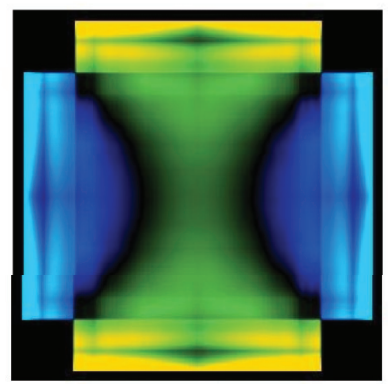

(c) $\beta \mu=0.07$

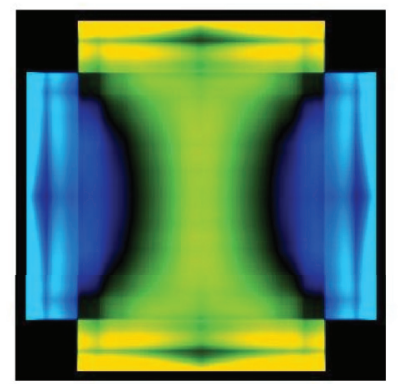

(d) $\beta \mu=0.31$

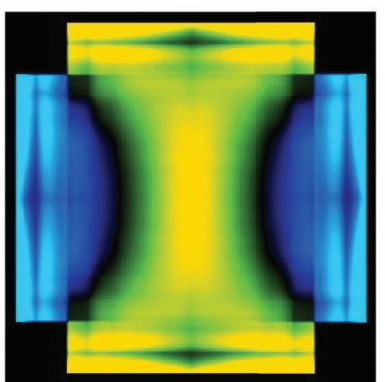

FIG. 11. (Color) Order-parameter false-color plots of confined structures in the case $\kappa=6$ and for $H=3 L$. Structures for different values of chemical potential (indicated on top of each panel) are shown. (a) Symmetric configuration (confined $I$ phase). (b)-(d) Symmetry-breaking configurations of increasing density. The confined $I \rightarrow N$ transition takes place between the (a) and (b) configurations. The case $\rho_{y}>\rho_{x}$ is represented in blue (dark grey), while the case $\rho_{y}<\rho_{x}$ is represented in yellow (light grey). 


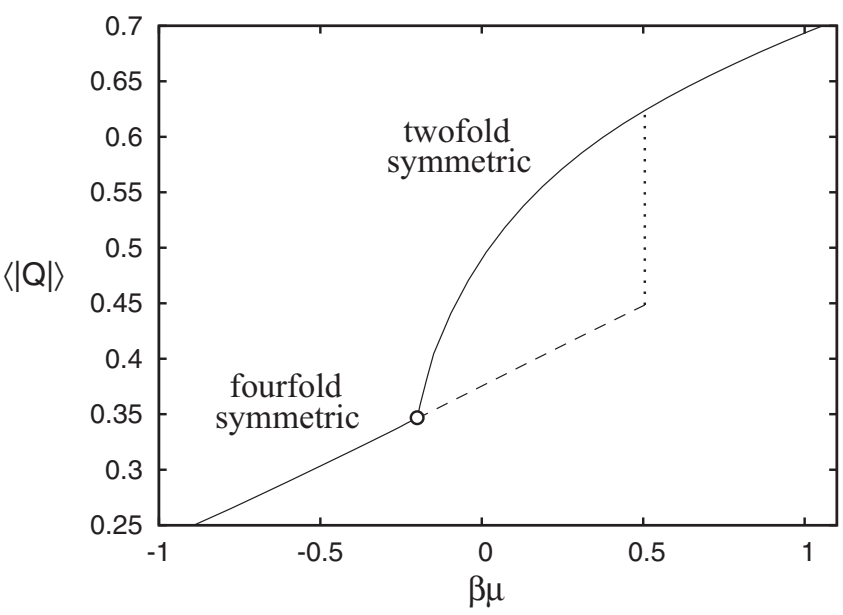

FIG. 13. Absolute value of the mean order parameter $\langle|Q|\rangle$ of the HR fluid with aspect ratio $\kappa=6$ as a function of chemical potential $\mu$ and for $H=3 L$. The solid curve corresponds to the stable (lowest grand potential) solution. The open circle is the bifurcation point separating the fourfold symmetric solution from the twofold symmetry-breaking solution. The dashed curve is the continuation of the (unstable) symmetric solution beyond the bifurcation point. The dotted vertical line indicates the terminal point for the symmetric solution and the jump to the symmetry break since the smectic layers become more delocalized in solution. Beyond the bifurcation point the stable solution is doubly degenerate (two equivalent solutions rotated by $90^{\circ}$ ).

of the bulk $I \rightarrow N$ transition as the cavity becomes larger. The transition is shown in Fig. 12 (the phase diagram in the $\mu$ - $H$ plane) as a continuous, nonmonotonic curve $\mu(H)$ in the neighborhood of the dashed line (the bulk value of the chemical potential $\left.\mu_{\text {bulk }}\right)$.

An interesting feature of the confined $I \rightarrow N$ transition is that it may occur below or above the bulk transition depending on $H$. The behavior of the confined $I \rightarrow N$ transition for very large cavities can be understood from the surface properties of the fluid near a single hard wall. Here we know that, as the continuous bulk $I \rightarrow N$ transition is approached from below, there is critical wetting by the $N$ phase of the wall- $I$ interface. In the cavity, close to but below the chemical potential of the bulk transition, a film of the almost-critical $N$ phase on each of the four walls should develop, causing orientational frustration in the central region of the cavity; this effect causes the symmetry-breaking mechanism to be postponed and the transition to occur above the bulk value $\mu_{I-N}$.

For a cavity size $H=H_{\text {min }} \simeq 1.6 L$ there is a minimum in the transition curve, followed by a maximum at larger cavities, $H_{\max } \simeq 4.0 \mathrm{~L}$. This feature is a consequence of particle size commensurate effects in the cavity. For $H \simeq 2.3 L$ [where $\mu(H) \simeq \mu_{I-N}$ ] the density maxima in the symmetric configuration occur near the four walls, but no longer near the cavity corners since not more than one particle can now be accommodated parallel and close to the walls. When $H \simeq 1.2 L$ [where again $\mu(H) \simeq \mu_{I-N}$ ] the density maximum is displaced at the center of the cavity, it is more difficult for particles to orient in the parallel configuration inside the cavity, and the transition line increases to very high chemical potential.

The symmetric solution continues to be a solution (from a numerical point of view) a bit beyond the bifurcation point. This is seen in Fig. 13, where the integrated absolute value of the order parameter,

$$
\langle|Q|\rangle=\frac{1}{A} \int_{A} d \boldsymbol{r}|Q(\boldsymbol{r})|,
$$

is plotted as a function of $\mu$ for $H=3 L$. The fourfold symmetric solution (dashed curve) exists beyond the bifurcation point (open circle), up to a point where it jumps to the twofold symmetry-breaking more stable solution.

Finally, the fact that the $I \rightarrow N$ transition in the confined fluid is a symmetry-breaking transition is confirmed by the fact that when the cavity is slightly deformed into a rectangular shape, the transition disappears altogether: All singularities in the grand potential vanish.

\section{Smectic commensurate transitions under confinement}

As in the previous cases, the high-density region of the phase diagram is dominated by commensurate transitions, but this time the transitions involve smectic phases with different numbers of layers. On increasing $H$ at fixed $\mu$, the system exhibits $\mathrm{Sm}_{n} \rightarrow \mathrm{Sm}_{n+1}$ first-order layering transitions between structures with numbers of layers differing by one (Fig. 12). These transitions are also obtained at fixed $H$ for increasing $\mu$ (a) $\mathrm{H}=7.3 \mathrm{~L}$

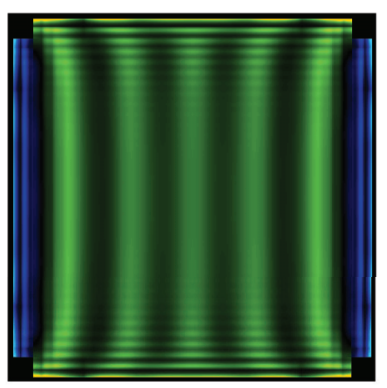

(b) $\mathrm{H}=7.2 \mathrm{~L}$

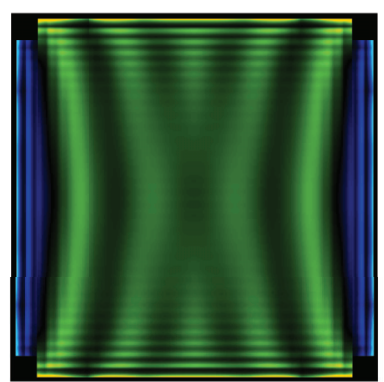

(c) $\mathrm{H}=6.8 \mathrm{~L}$

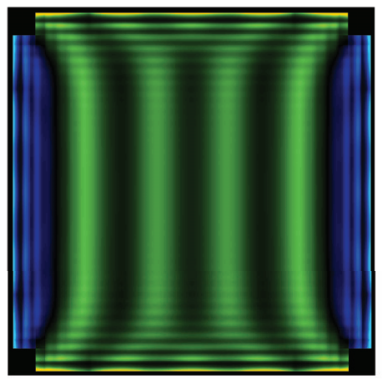

(d) $\mathrm{H}=6.2 \mathrm{~L}$

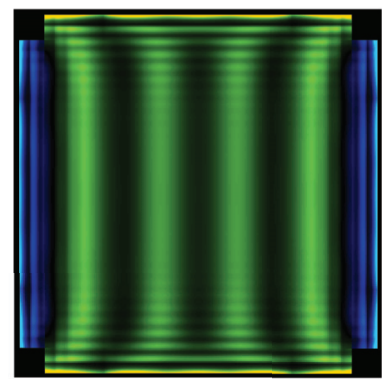

FIG. 14. (Color) Density contour plots of confined structures in the case $\kappa=6$ at high chemical potential $\beta \mu=2.336$. Different cavity sizes from $H=7.3 \mathrm{~L}$ down to $6.2 \mathrm{~L}$ are shown. These structures correspond to the $\mathrm{Sm}_{5} \rightarrow \mathrm{Sm}_{4}$ transition. Note that it is actually the product $Q(\boldsymbol{r}) \rho(\boldsymbol{r})$ that is plotted in the graphs, with the case $\rho_{y}>\rho_{x}$ represented in blue (layered structure next to vertical walls) and the case $\rho_{y}<\rho_{x}$ represented in green. 
and end in critical points at chemical potentials that lie below the bulk value $\mu_{N \text {-Sm }}$ for the $N \rightarrow$ Sm transition (dotted horizontal line in the figure), but that approach $\mu_{N \text {-Sm }}$ as $H \rightarrow \infty$. This is a capillary effect: Smectic layers are easily stabilized in the cavity since the order parameter rapidly saturates once the symmetry-breaking nematic phase is established. Density plots for the transition between $\mathrm{Sm}_{4}$ and $\mathrm{Sm}_{5}$ are shown in Fig. 14 at fixed $\mu$ for decreasing $H$ (i.e., the system loses one smectic layer). Again, since the orientational order parameter is almost saturated, only the translational degrees of freedom are important; the commensurate mechanism is effective along the direction perpendicular to the layers and involves the distance between the walls parallel to the layers (vertical walls in the figure). The other two walls (perpendicular to the layers) induce increased ordering next to the walls. In contrast to the previous case, where the new column was nucleated in the central region and the surface structure remained unaltered, in this case the layer-growth mechanism involves defects that begin or end at the walls perpendicular to the layers. In the case of layer growth, two defects are created at the two walls, which then propagate to the center of the cavity and give rise to a new smectic layer. In the case where one layer disappears, the defects are formed at the center and then migrate to the walls. This is the case in Fig. 14, which corresponds to the $\mathrm{Sm}_{5} \rightarrow \mathrm{Sm}_{4}$ transition as $H$ is decreased (see structures for $H=7.2 L$ and $6.8 L$ ). Note that the structure next to the vertical walls grows a bit as the transition takes place. Again this behavior may be representative of the nucleation processes involving a change of one smectic layer. Figures 14(a) and 14(b) correspond to the metastable $\mathrm{Sm}_{5}$ phase; in Figs. 14(c) and 14(d) the system is already in the stable free-energy branch of the $\mathrm{Sm}_{4}$ phase. A film of the evolution of the fluid as the cavity size $H$ is varied can be found in [38].

\section{LIMITATIONS OF THE MODEL AND THE $I \rightarrow N$ TRANSITION}

Our theory has a gross built-in approximation, namely, particle orientations are restricted to only two perpendicular directions and this will certainly misrepresent particle configurations where the sides of two particles are at an angle $0^{\circ}<\varphi<90^{\circ}$. By contrast, spatial correlations of parallel $\left(\varphi=0^{\circ}\right)$ and perpendicular $\left(\varphi=90^{\circ}\right)$ configurations are probably very well represented. Since the high-density bulk and confined phases are mostly composed of particles in parallel or perpendicular configurations, the predictions of the theory on the bulk and surface properties in this regime are qualitatively, if not quantitatively, correct. This includes the prediction that, in bulk, the Col or Sm phases are stable up to very high values of packing fraction and that the Col $(\mathrm{Sm})$ phase is favored for low (high) particle aspect ratios. Phenomena involving the isotropic and nematic phases, although qualitatively correct, may be prone to larger errors. For example, the prediction of the suppression of the $N$ phase in bulk for low aspect ratios is correct, but the value of aspect ratio for which this occurs may not be very accurate, as well as the location of the $I \rightarrow N$ transition itself. An Onsager-like theory, such as the one used in $[39,40]$, would give more realistic predictions in this low-density region, but will provide unreliable predictions in the high-density limit, which is the goal of our study.

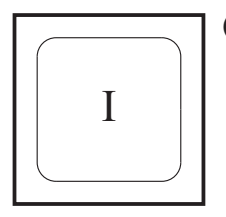

(a)

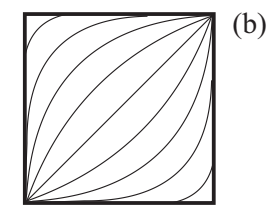

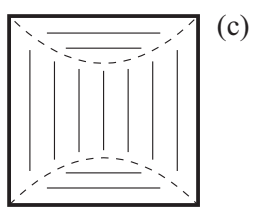

FIG. 15. Possible phases of the HR fluid confined in a square cavity in the nematic regime: (a) isotropic phase with a film of nematic adsorbed on the inner walls; (b) distorted nematic phase with two distinct pairs of defects, the components of each pair at opposite corners of the cavity; and (c) nematic phase with two opposing defect curves.

Although our study is focused on the high-density region of the phase diagram, a side aspect is the nature and structure of the confined nematic phase (in cases where this phase is stable in bulk) and our model still could be useful well inside the nematic region. In our study we have always observed that the isotropic configuration [Fig. 15(a)] changes to a nematic configuration where a region of uniform director is bounded by two smaller regions with the director at perpendicular directions [the structure discussed in Sec. IV C1 and represented in Figs. 11(b)-11(d)]. This configuration, with its associated director field depicted schematically in Fig. 15(c), satisfies the favored orientation at the four walls with no elastic free-energy cost, but incurs a free energy due to the presence of two curved interfaces (domain walls) across which the director changes by $90^{\circ}$. Experiments on quasimonolayers of granular cylinders [24] show the existence of a structure with two pairs of distinct defects located on opposite corners of the square cavity [Fig. 15(b)]. In this structure the surface orientation is also satisfied, but there are four point defects at the corners and some distortion of the director field, with the corresponding elastic free energy. The stable equilibrium configuration will result, in a more realistic model, from a balance between defect and elastic-distortion free energies. Since elastic constants increase with density (see, e.g., [40] for calculations on a two-dimensional model of hard discorectangles), the structure depicted in Fig. 15(c) is expected to ultimately become stable at high packing fraction. Ongoing Monte Carlo simulations [41] of the HR fluid confined in a square cavity show that on increasing the density of the fluid, the isotropic phase becomes nematic following the phase sequence plotted in Fig. 15. The fact that the intermediate structure depicted in Fig. 15(b) is not observed in our model is due to the simplistic representation of particle orientations, which, as mentioned in Sec. III, precludes configurations where the director is distorted uniformly (i.e., without a region of discontinuity).

A way to systematically improve the present restricted model without dealing with the more numerically demanding free-orientation model is to include more species in the set of restricted orientations. For example, by including species with orientations at $45^{\circ}$ and $135^{\circ}$ (and their equivalent orientations at $225^{\circ}$ and $335^{\circ}$ ), particles could, for some external conditions, be highly oriented along the cavity diagonal and this constitutes a necessary (not sufficient) condition to stabilize the structure shown in Fig. 1(e). Testing this hypothesis is difficult since the derivation of a fundamental-measure density functional for four species of HR would require severe approximations that would certainly affect the accurate 
description of particle correlations. As an initial approach we have checked that a scaled-particle theory (SPT) description for the restricted-orientation model with four species gives a bulk $I \rightarrow N$ transition curve located above the one obtained from the two-species SPT, which is the uniform limit of the present model (these results are not shown here). We mention that, as a bonus, the four-species SPT allows us to describe the nematic phase with tetratic (fourfold) symmetry, a phase that is also predicted by the free-orientation models. This approach might be worthwhile to follow in the context of future work on the structure of confined fluids.

\section{CONCLUSION}

In summary, we have studied the structure of a fluid of hard rectangles inside a square cavity using a fundamental-measure version of density-functional theory. Due to the restrictedorientation approximation inherent to the model, the prediction of the suppression of the $N$ phase in bulk for low aspect ratios is correct, but the value of aspect ratio for which this occurs may not be very accurate, as well as the location of the $I \rightarrow N$ symmetry-breaking transition itself. However, the prediction that, in bulk, the Col or Sm phases are stable up to very high values of packing fraction and that the Col (Sm) phase is favored for low (high) aspect ratios should be qualitatively, if not quantitatively, correct.

In the confined system the model predicts the occurrence of commensurate transitions between structures that differ in one unit cell (either the Col, Sm, or Cry phase). The symmetrybreaking $I \rightarrow N$ transition is obtained as a continuation of the bulk transition in the confined system whenever there is a stable bulk $N$ phase and results from the square symmetry of the cavity; in rectangular, even slightly nonsquare, cavities, the $I \rightarrow N$ transition is suppressed altogether. The phase boundary of the transition has a complicated, nonmonotonic behavior with respect to cavity size; this behavior is probably correct for small cavities where, due to the square symmetry of the cavity, parallel and perpendicular orientations may be much more probable and the theory should be more accurate.

The general scenario that emerges from the present work is the following. In a severely restricted geometry such as the square cavity, a liquid-crystal fluid is subject to several competing mechanisms, i.e., surface interaction causing frustration, elasticity, and defect formation, the competition of which causes a complex behavior in the confined nematic system. This problem has been studied several times in the past. Since our model can predict the stability of nonuniform bulk (Sm, Col, and Cry) phases, we have been able to extend these studies to incorporate the effect of periodicity and the commensurate problems associated with a periodic bulk phase in a confined geometry. Capillarity, surface-generated frustration, and commensurate effects all work together to create complex phase behavior in the confined fluid.

Finally, several lines of future research may be worth pursuing. For example, a mixture inside the cavity adds a further mechanism in the way of demixing; the connection of bulk demixing and surface segregation with confinement, capillarity, and frustration may add up to the richness in the phenomenology, with possible implications for experiments on granular mixtures of particles. In contrast, simulation studies of this system would be very welcome [41] since they may confirm the behavior implied by the present theoretical model. Also, the present model can be modified a bit to adapt it to a cylindrical geometry by introducing periodic boundary conditions in one of the directions; this model could be useful to understand the bacterial growth mechanism recently suggested by Nelson and Amir [42].

\section{ACKNOWLEDGMENTS}

We acknowledge financial support from Program No. MODELICO-CM/S2009ESP-1691 at Comunidad Autónoma de Madrid, Spain, and Programs No. FIS2010-22047-C01 and No. FIS2010-22047-C04 at MINECO, Spain.
[1] T. J. Sluckin and A. Poniewierski, Mol. Cryst. Liq. Cryst. 179, 349 (1990).

[2] P. Pieranski, L. Strzelecki, and B. Pansu, Phys. Rev. Lett. 50, 900 (1983).

[3] G. Navascués and P. Tarazona, Mol. Phys. 62, 497 (1987).

[4] D. Chaudhuri and S. Sengupta, Phys. Rev. Lett. 93, 115702 (2004).

[5] D. de las Heras, E. Velasco, and L. Mederos, Phys. Rev. Lett. 94, 017801 (2005).

[6] D. de las Heras, E. Velasco, and L. Mederos, Phys. Rev. E 74, 011709 (2006).

[7] V. Babin, A. Ciach, and M. Tasinkevych, J. Chem. Phys. 114, 9585 (2001).

[8] M. Tasinkevych and A. Ciach, Phys. Rev. E 72, 061704 (2005).

[9] T. Geisinger, M. Muller, and K. Binder, J. Chem. Phys. 111, 5241 (1999).

[10] Y. Martínez-Ratón, Phys. Rev. E 75, 051708 (2007).

[11] N. Schopohl and T. J. Sluckin, Phys. Rev. Lett. 59, 2582 (1987).
[12] P. Palffy-Muhoray, E. C. Garland, and J. R. Kelly, Liq. Cryst. 16, 713 (1994).

[13] H. G. Galabova, N. Kothekar, and D. W. Allender, Liq. Cryst. 23, 803 (1997).

[14] A. Šarlah and S. Žumer, Phys. Rev. E 60, 1821 (1999).

[15] D. de las Heras, L. Mederos, and E. Velasco, Phys. Rev. E 79, 011712 (2009).

[16] P. I. C. Teixeira, F. Barmes, C. Anquetil-Deck, and D. J. Cleaver, Phys. Rev. E 79, 011709 (2009).

[17] C. Chiccoli, P. Pasini, A. Šarlah, C. Zannoni, and S. Žumer, Phys. Rev. E 67, 050703(R) (2003).

[18] C. Chiccoli, S. P. Gouripeddi, P. Pasini, R. P. N. Murthy, V. S. S. Sastry, and C. Zannoni, Mol. Cryst. Liq. Cryst. 500, 118 (2009).

[19] R. G. Marguta, Y. Martínez-Ratón, N. G. Almarza, and E. Velasco, Phys. Rev. E 83, 041701 (2011).

[20] D. de las Heras and E. Velasco (unpublished).

[21] H. Löwen, J. Phys.: Condens. Matter 22, 364105 (2010).

[22] I. S. Aranson and L. S. Tsimring, Rev. Mod. Phys. 78, 641 (2006). 
[23] V. Narayan, N. Menon, and S. Ramaswamy, J. Stat. Mech. (2006) P01005.

[24] J. Galanis, D. Harries, D. L. Sackett, W. Losert, and R. Nossal, Phys. Rev. Lett. 96, 028002 (2006).

[25] H. Schlacken, H.-J. Mogel, and P. Schiller, Mol. Phys. 93, 777 (1998).

[26] Y. Martínez-Ratón, E. Velasco, and L. Mederos, J. Chem. Phys. 122, 064903 (2005).

[27] Computational Methods in Science and Technology, edited by K. W. Wojciechowski and D. Frenkel (Science Publishers OWN, Poznań, 2004), pp. 10 and 235.

[28] A. Donev, J. Burton, F. H. Stillinger, and S. Torquato, Phys. Rev. B 73, 054109 (2006).

[29] K. Zhao, C. Harrison, D. Huse, W. B. Russel, and P. M. Chaikin, Phys. Rev. E 76, 040401 (2007).

[30] J. Geng and J. V. Selinger, Phys. Rev. E 80, 011707 (2009).

[31] Y. Martínez-Ratón and E. Velasco, Phys. Rev. E 79, 011711 (2009).

[32] Y. Martínez-Ratón, E. Velasco, and L. Mederos, J. Chem. Phys. 125, 014501 (2006).
[33] W. G. Hoover, C. G. Hoover, and M. N. Bannerman, J. Stat. Phys. 136, 715 (2009).

[34] K. Zhao, R. Bruisnma, and T. G. Mason, Proc. Natl. Acad. Sci. U.S.A. 108, 2684 (2011).

[35] S. Belli, M. Dijkstra, and R. van Roij, J. Chem. Phys. 137, 124506 (2012).

[36] D. A. Triplett and K. A. Fichthorn, Phys. Rev. E 77, 011707 (2008).

[37] J. A. Cuesta and Y. Martínez-Ratón, Phys. Rev. Lett. 78, 3681 (1997).

[38] See Supplemental Material at http://link.aps.org/supplemental/ 10.1103/PhysRevE.88.032506 for a movie that shows an example of commensurate transitions involving different phases.

[39] D. de las Heras, E. Velasco, and L. Mederos, Phys. Rev. E 79, 061703 (2009).

[40] D. de las Heras, L. Mederos, and E. Velasco, Liq. Cryst. 37, 45 (2009).

[41] D. de las Heras (private communication).

[42] D. R. Nelson and A. Amir, arXiv:1303.5896v1. 